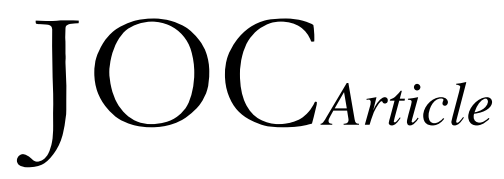

\title{
Dynamic Chirality in Donor-Acceptor Pretzelanes
}

Y. Liu, S. A. Vignon, X. Zhang, P. A. Bonvallet, S. I. Khan, K. N. Houk, J. F. Stoddart*

California NanoSystems Institute and Department of Chemistry and Biochemistry, University of California, Los Angeles, 405 Hilgard Avenue, Los Angeles, CA, 90095 1569, USA

stoddart@chem.ucla.edu

Received July XX, 2005

\section{Supporting Information}

\section{Contents}

General Methods S2

NMR Spectra

Figure S1 S3

Figure S2 S4

Figure S3 .S5

Figure S4 .S6

Figure S5 S7

Figure S6 S8

References 
General Methods: Reagents were purchased or synthesized as described. Solvents were purified according to literature procedures. ${ }^{1}$ Thin-layer chromatography (TLC) was carried out using aluminum sheets, precoated with silica gel 60F. The plates were inspected by UV-light, prior to development with iodine vapor. Melting points are uncorrected. Proton and carbon nuclear magnetic resonance spectra $\left({ }^{1} \mathrm{H}-\mathrm{NMR}\right.$ and ${ }^{13} \mathrm{C}$ NMR) were recorded using the deuterated solvent as lock and the residual protiated solvent as internal standard. All chemical shifts are quoted using the $\delta$ scale, and all coupling constants $(J)$ are expressed in Hertz $(\mathrm{Hz})$. Electrospray mass spectra (ESI-MS) were measured on a triple focusing mass spectrometer.

NMR Spectra: What follows are NMR spectra of selected intermediates in the syntheses of $\mathbf{2} \cdot 4 \mathrm{PF}_{6}$ and $\mathbf{3} \cdot 4 \mathrm{PF}_{6}$. 


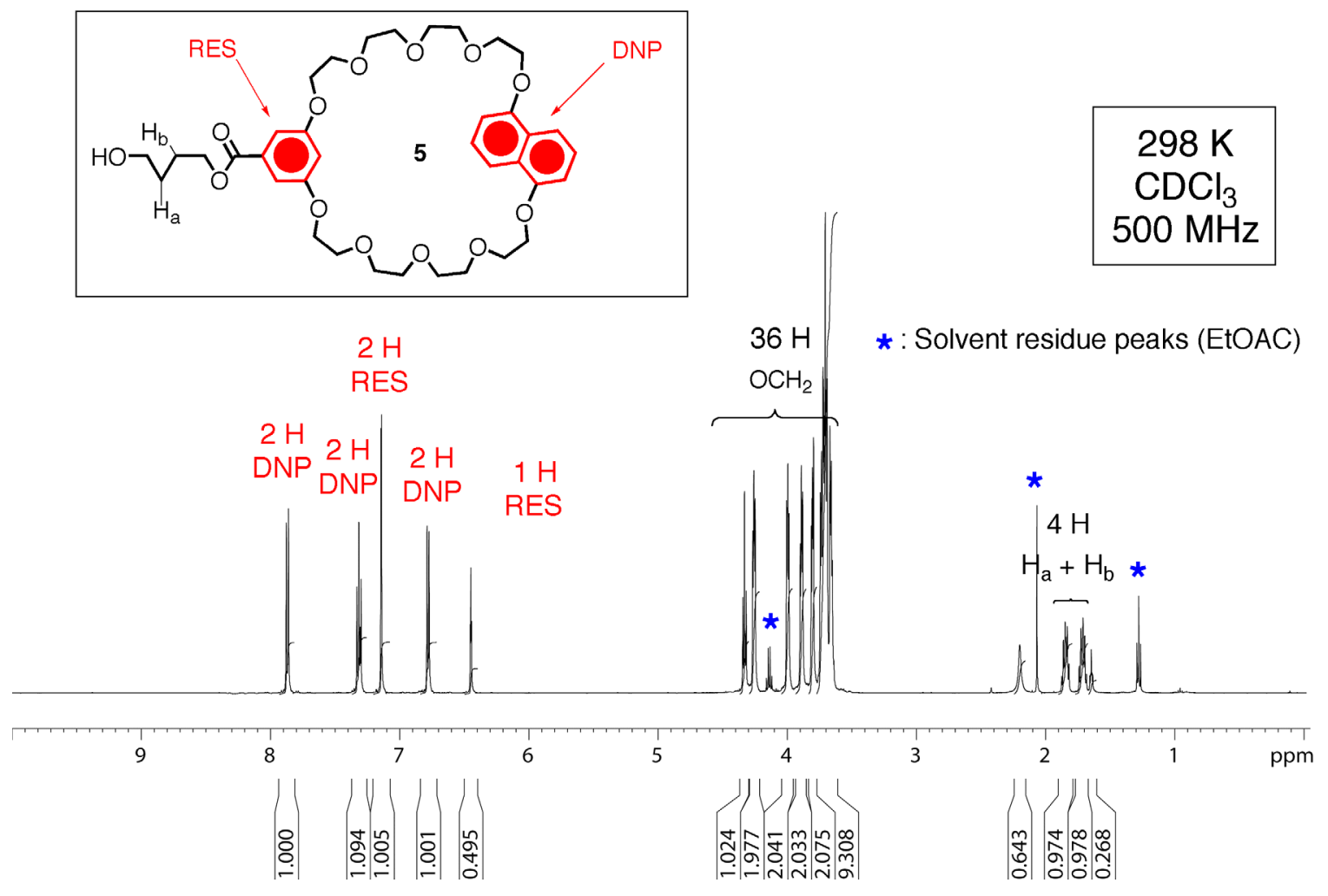

Figure S1. ${ }^{1} \mathrm{H}$ NMR spectrum of 7 . 


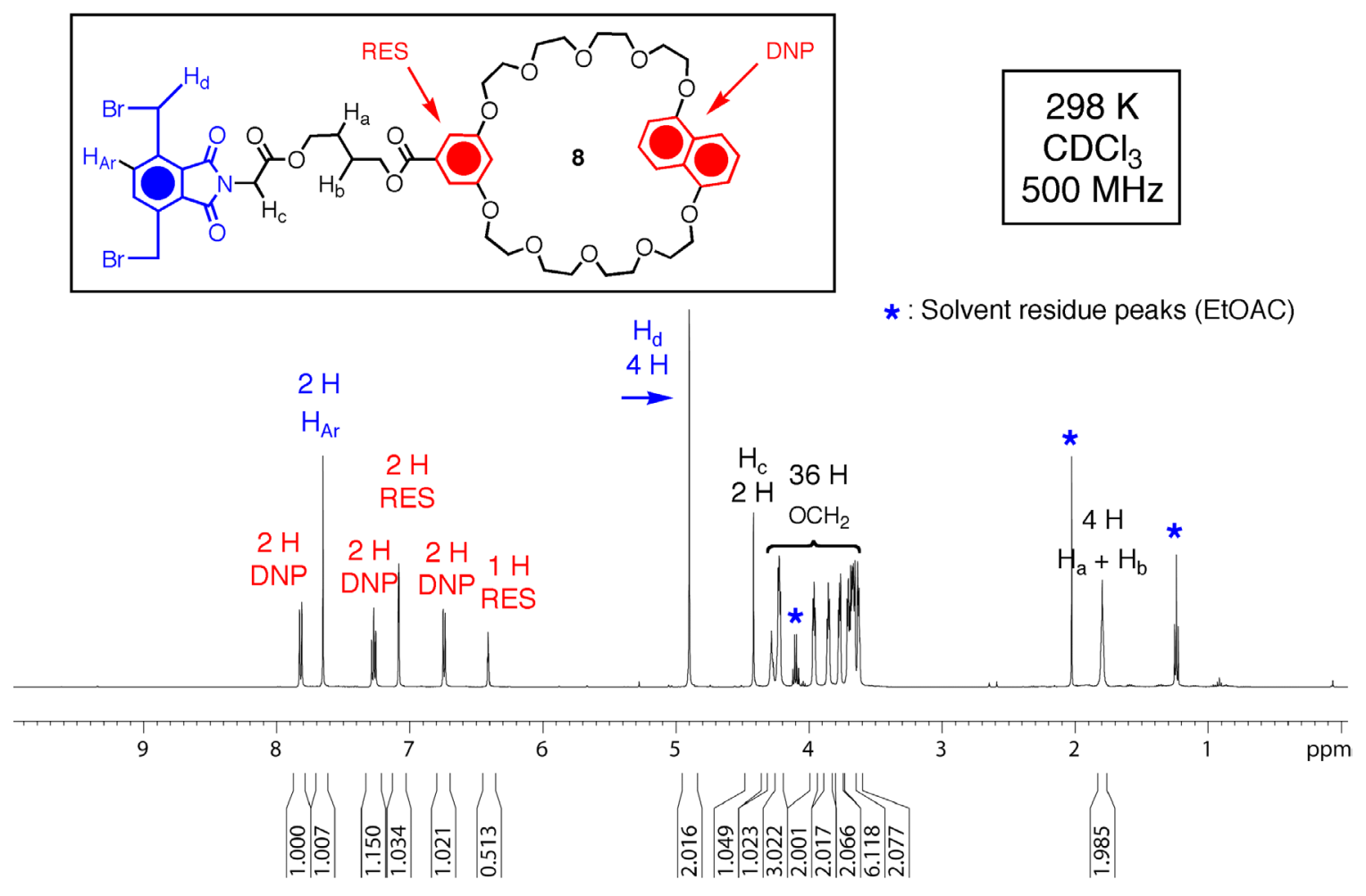

Figure S2. ${ }^{1} \mathrm{H}$ NMR spectrum of $\mathbf{1 0}$. 


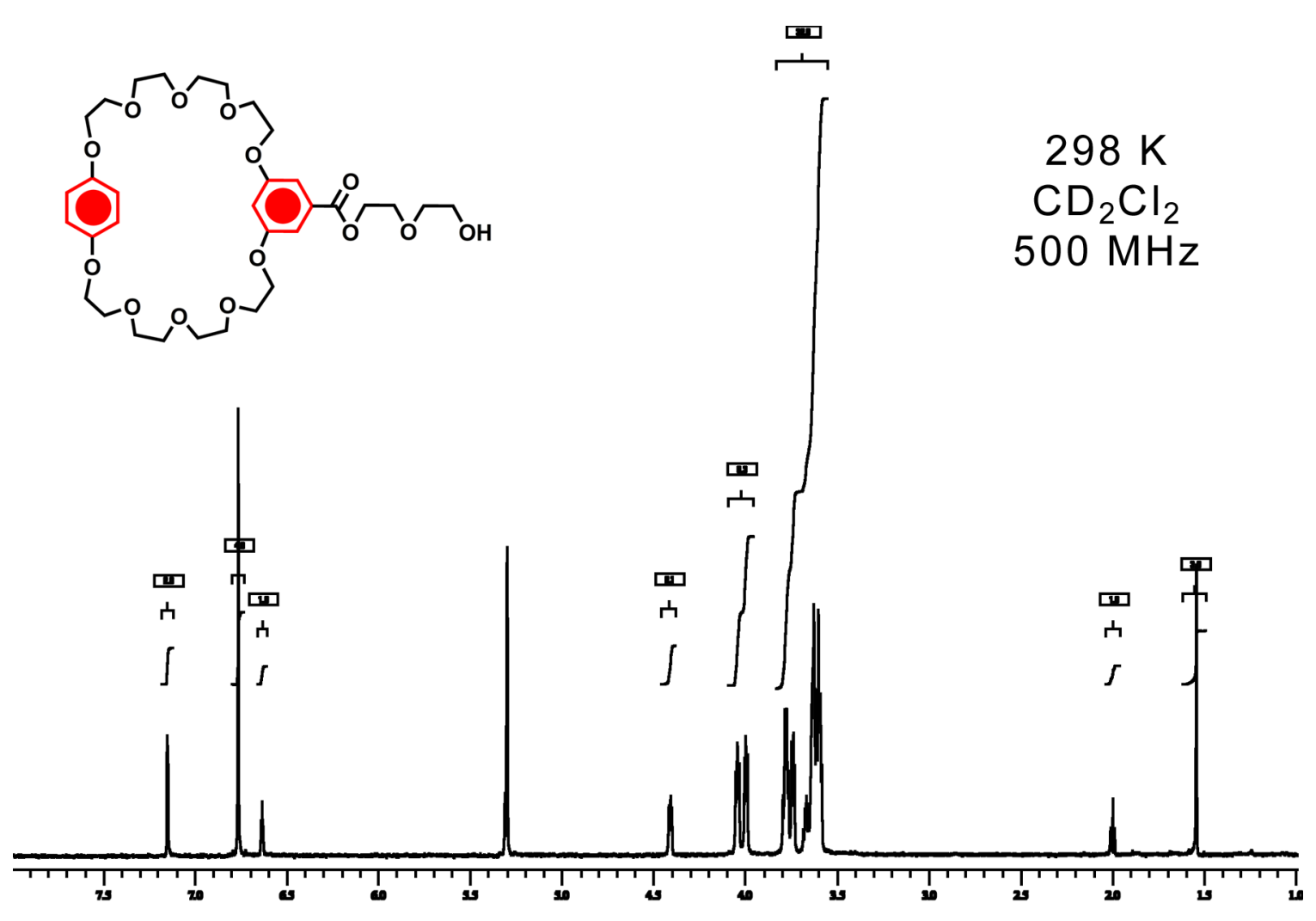

Figure S3. ${ }^{1} \mathrm{H}$ NMR spectrum of 13. 


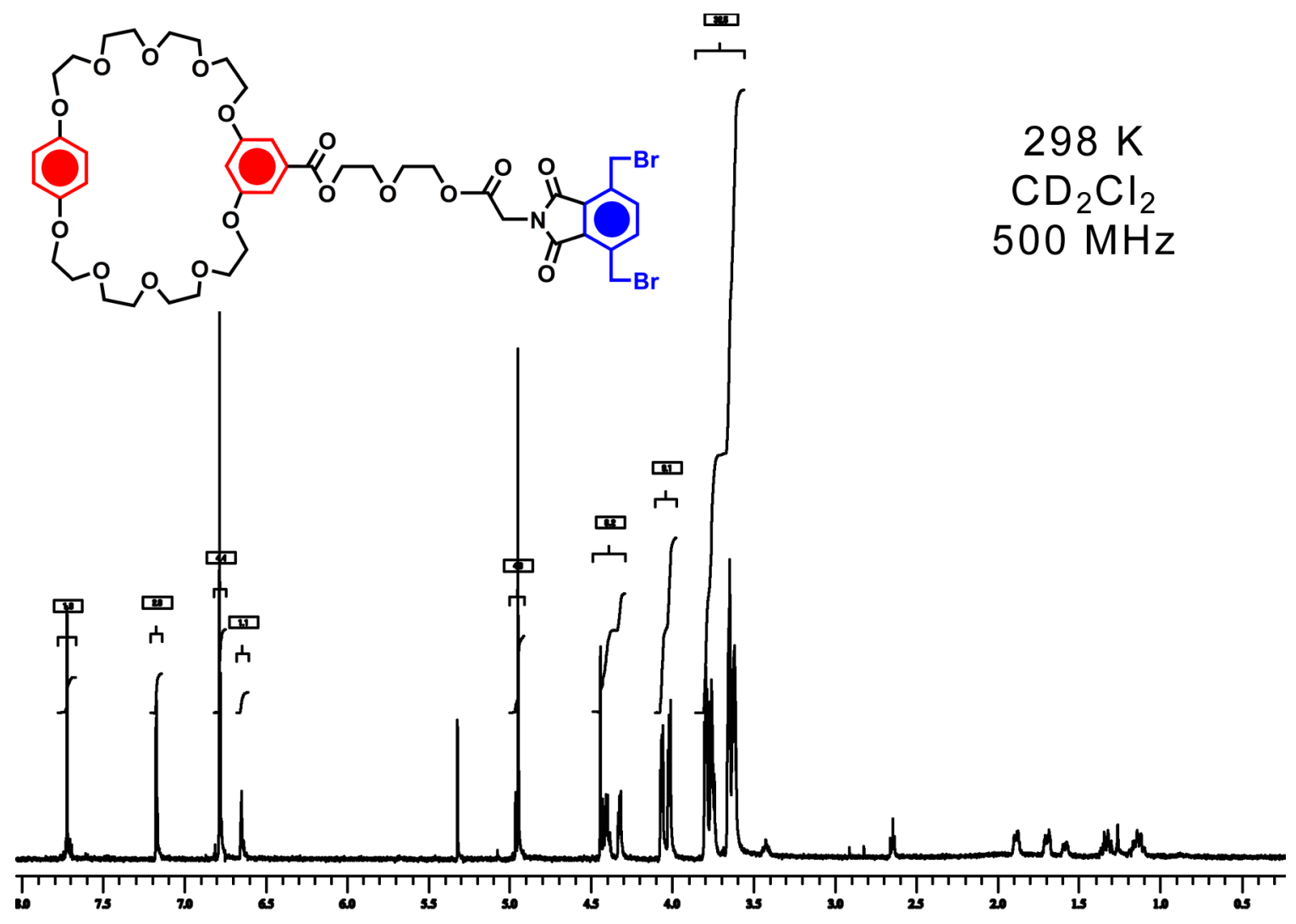

Figure S4. ${ }^{1} \mathrm{H}$ NMR spectrum of 14. 


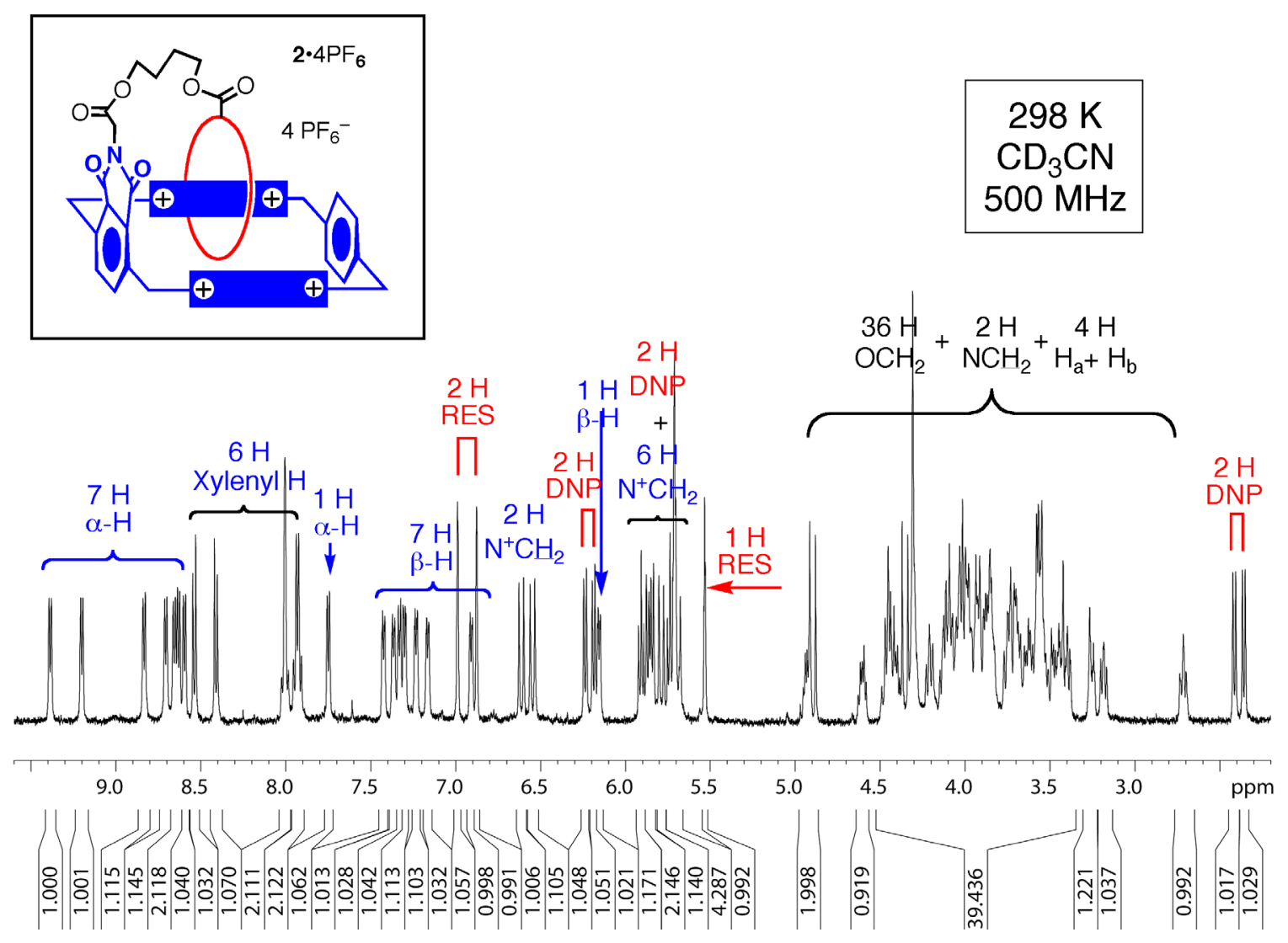

Figure S5. ${ }^{1} \mathrm{H}$ NMR spectrum of $\mathbf{2} \bullet 4 \mathrm{PF}_{6}$. 

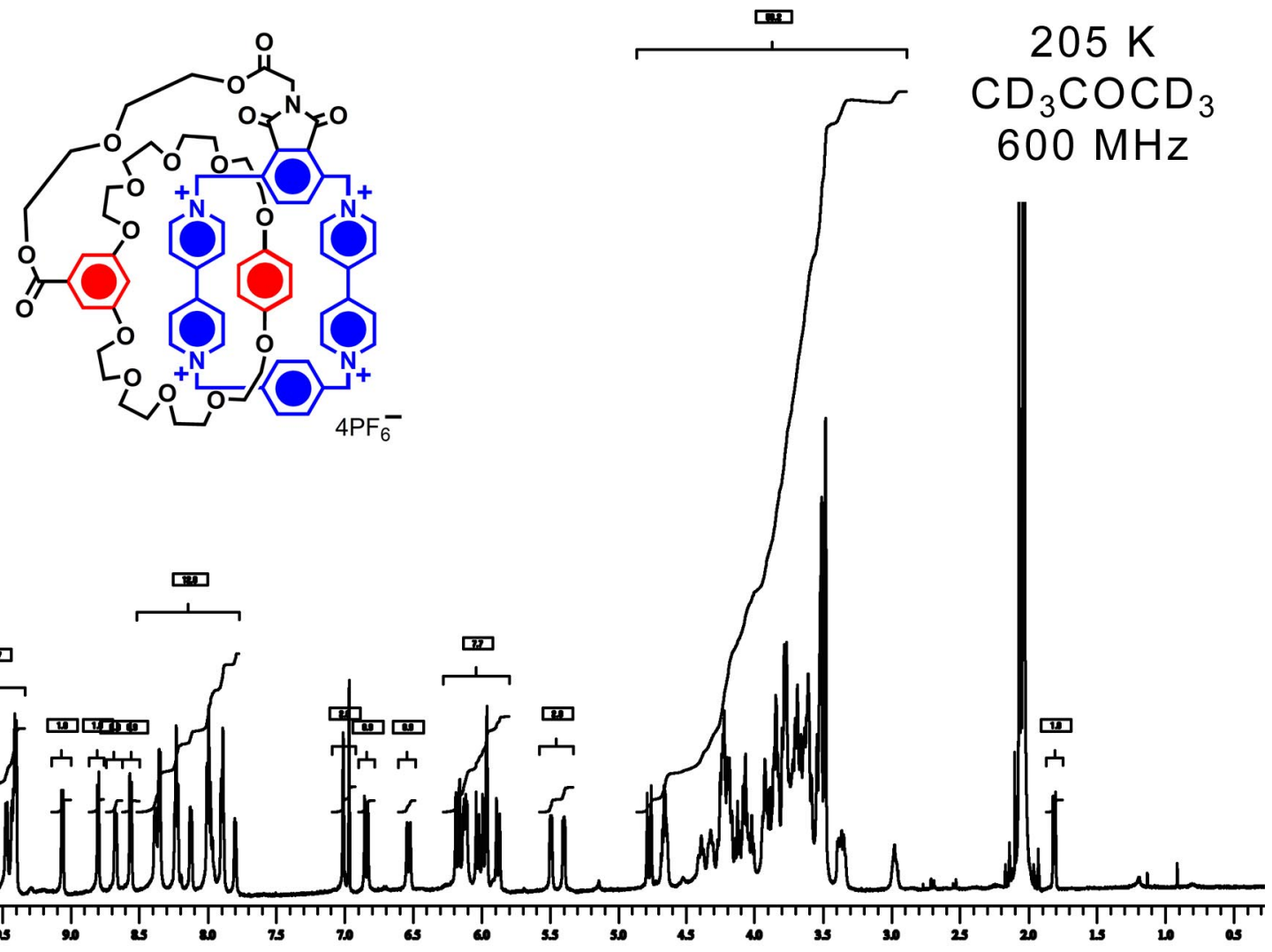

Figure S6. ${ }^{1} \mathrm{H}$ NMR spectrum of $\mathbf{3} \cdot 4 \mathrm{PF}_{6}$. 


\section{References:}

1 Perrin, D. D.; Armarego, W. F. L. Purification of Laboratory Chemicals; Pergamon, Oxford, 1989. 